\title{
TEMA 6-2015: TROMBOEMBOLISMO PULMONAR: FISIOPATOLOGÍA Y DIAGNÓSTICO.
}
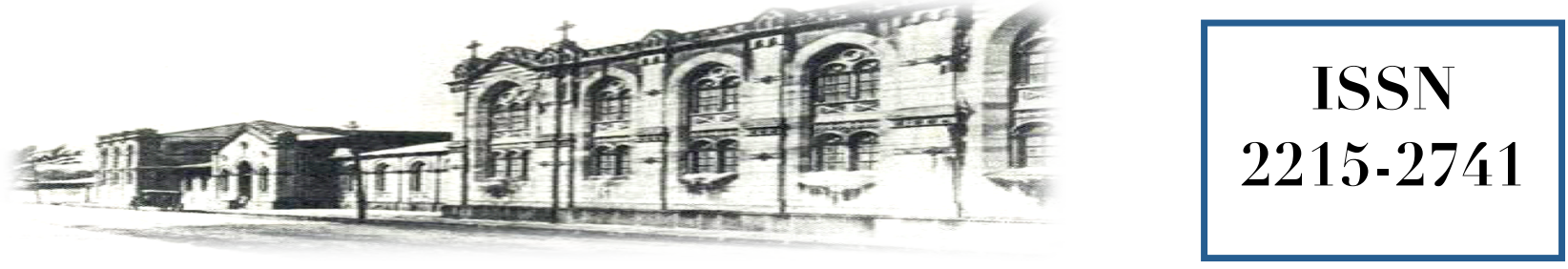

Hospital San quan de Dias, San José, Costa Rica. Fundado en 1845

Recibido:

$08 / 03 / 2015$

Aceptado:

$25 / 03 / 2015$

Carlos Fernando Estrada Garzona ${ }^{1}$ Andrés Francisco Garzona Navas ${ }^{2}$

\footnotetext{
${ }^{1}$ Médico-Cirujano. Profesor del Departamento de Farmacología. Escuela de Medicina. Universidad de Costa Rica. Correo electrónico; drestradag@gmail.com

${ }^{2}$ Médico-Cirujano. Universidad de Costa Rica. Clínica Quirúrgica la Inmaculada. Correo electrónico: andresgarzona@gmail.com
}

\section{RESUMEN}

El tromboembolismo pulmonar (TEP) es un evento clínico frecuente asociado a importante morbi-mortalidad que varía según características del evento y del paciente. Múltiples factores de riesgo han sido identificados con distinto grado de asociación con TEP, destacando el cáncer, las enfermedades infecciosas e inflamatorias, trombosis previas, entre otros. El diagnóstico de TEP debe basarse en una estrategia clínica lógica, individualizando al paciente según su grado de probabilidad, tomando en cuenta los hallazgos clínicos, de laboratorio y gabinete, analizando diferentes ventajas y desventajas de cada una de las herramientas diagnósticas. Este artículo pretende brindar una revisión de la epidemiología, fisiopatología y diagnóstico del TEP.

\section{PALABRAS CLAVE}

Tromboembolismo Pulmonar, fisiopatología, factores de riesgo, diagnóstico.

\section{ABSTRACT}

Pulmonary embolism (PE) is a common clinical event associated with significant morbidity and mortality that varies according to characteristics of the event and the patient. Multiple risk factors have been identified with a different degree of association; including cancer, infectious and inflammatory diseases, and previous thrombotic events, among others. PE diagnosis should be based on a logical clinical strategy, individualized to the patient according to their probability, considering clinical, laboratory and imaging 
findings, analyzing the different advantages and disadvantages of each diagnostic tool. This article aims to provide a review of the epidemiology, pathophysiology and diagnosis of PE.

\section{KEY WORDS}

Pulmonary Embolism, risk factors, pathophysiology, diagnosis.

\section{INTRODUCCIÓN}

El tromboembolismo pulmonar (TEP) es una causa frecuente de hospitalización, asociada a importante morbilidad y mortalidad en múltiples contextos clínicos ${ }^{(1)}$. Este trabajo pretende brindar una revisión de la epidemiología, fisiopatología y diagnóstico del TEP.

\section{DISCUSIÓN}

\section{Definición de Embolismo Pulmonar}

La incidencia de complicaciones asociadas al TEP debe contemplar la presencia de comorbilidades del paciente y su efecto sobre la evolución clínica posterior al embolismo. De esta manera, conviene establecer la definición del TEP en tres formas de presentación ${ }^{(1)}$ :

- Embolismo masivo

- Embolismo submasivo

- Embolismo de bajo riesgo

El embolismo pulmonar masivo se caracteriza por la presencia de un cuadro agudo con hipotensión sostenida (presión arterial sistólica menor a $90 \mathrm{mmHg}$ por al menos 15 minutos o que amerita soporte inotrópico o vasopresor), que no es atribuible a hipovolemia, sepsis, disfunción ventricular izquierda o arritmia; ausencia de pulso o bradicardia profunda persistente con signos o síntomas de shock) ${ }^{(1)}$.

La mortalidad intrahospitalaria del paciente con TEP agudo sin datos de inestabilidad hemodinámica pasa de $8.1-14.7 \%$ a valores de $25-52.4 \%$ al evidenciarse alguno de los criterios contemplados en la definición de embolismo $\operatorname{masivo}^{(1-3)}$.
Un embolismo pulmonar agudo sin hipotensión sistémica (presión arterial sistólica $>90 \mathrm{mmHg}$ ) pero con datos de disfunción ventricular derecha o necrosis miocárdica establece el diagnóstico de TEP submasivo ${ }^{(1)}$. La necrosis miocárdica se manifiesta por la elevación de troponina I $(>0.4$ $\mathrm{ng} / \mathrm{ml})$ o troponina $\mathrm{T}(>0.1 \mathrm{ng} / \mathrm{ml})$; mientras que la disfunción ventricular derecha se determina al documentarse al menos 1 de los siguientes criterios $^{(1)}$ :

- Ecocardiograma con dilatación ventricular derecha (diámetro 4 cámaras apical entre el diámetro ventricular izquierdo $>0.9$ ) o disfunción sistólica.

- Tomografía axial con dilatación ventricular derecha (diámetro 4 cámaras apical entre el diámetro ventricular izquierdo $>0.9$ ).

- Elevación de péptido natriurético cerebral (BNP) (>90 pg/ml).

- Elevación del N-terminal pro-BNP (>500 $\mathrm{pg} / \mathrm{ml})$.

- Electrocardiograma con bloqueo de rama derecha de novo; elevación o depresión anteroseptal del segmento ST; o inversión de la onda $\mathrm{T}$ anteroseptal.

Por otro lado, los pacientes con TEP agudo normotensos y sin datos de disfunción ventricular derecha $\mathrm{y} / \mathrm{o}$ necrosis miocárdica se pueden definir como embolismo pulmonar de bajo riesgo y con pronóstico mejor que los dos grupos anteriores $^{(1)}$.

\section{Trombosis venosa. Fisiopatología}

El concepto de trombosis venosa (TV) nace como eje fundamental del fenómeno fisiopatológico del embolismo pulmonar a partir de los postulados de Rudolf Virchow en 1856, quien establece que el daño a la pared del vaso sanguíneo, la alteración del flujo sanguíneo y un estado de hipercoagulabiilidad son las causas principales de la formación del trombo venoso ${ }^{(4)}$.

El sitio más frecuente de formación del trombo se ubica en las válvulas venosas de los vasos de la región sural que migran hacia territorios más proximales como la región poplítea, de donde se hace más probable la embolización ${ }^{(5,6)}$; la obstrucción venosa generada por este trombo se recanaliza en un periodo de 3 meses en $50 \%$ de los pacientes y en aquellos que se produce incompetencia valvular residual se identifica edema tardío como hallazgo clínico ${ }^{(4,7)}$. 


\section{Epidemiología. Embolismo Pulmonar.}

La incidencia y prevalencia del embolismo pulmonar debe contextualizarse en tres escenarios clínicos: paciente ambulatorio, hospitalizado y la paciente embarazada ${ }^{(6,7)}$.

La incidencia anual de tromboembolismo venoso en población estadounidense e inglesa se ha documentado en 71-74.5 casos por 100.000 pacientes-año en cohortes de estudios comunitarios y de bases de datos poblacionales $^{(8,9)}$, mientras que en una cohorte francesa es ligeramente menor (60 casos por 100.000 pacientes año ${ }^{(10)}$. En población asiática, las cohortes publicadas con datos de Korea y Singapur reportan datos aún más bajos de incidencia, con valores de 7-15 casos por 100.000 pacientes-año ${ }^{(11,12)}$.

En el paciente hospitalizado, la incidencia de TEP es mucho menor incluso en las cohortes mencionadas anteriormente; en la comunidad de Olmsted, Estados Unidos de América, la incidencia de TEP en el paciente hospitalizado es de 9.6 casos por 100.000 pacientes-año ${ }^{(8)}$. Sin embargo, la incidencia en cohortes con periodos de seguimiento mayor, reportada en porcentaje y sin estandarizar por el periodo de seguimiento, corresponden a valores menores al $1 \%(0.1$ $0.4 \%$ ) en cohortes tanto de estadounidenses como asiáticas $^{(13,14)}$

La incidencia de embolismo pulmonar en la paciente periparto aumenta al compararlo con los valores reportados para el paciente hospitalizado, con valores de $0.12-0.18$ casos por 1.000 partosaño ${ }^{(15)}$ y la incidencia acumulada alcanza valores de 3.24 casos de TEP por cada 1000 partosaño ${ }^{(16)}$.

La mortalidad anual asociada a TEP agudo se ha reportado en valores de 300.000 casos en Estados Unidos y con tasas de 2.7-12.8 muertes por cada 100.000 pacientes-año en Europa ${ }^{(17-19)}$.

Dado que en el paciente con TEP se logra identificar la presencia de trombosis venosa profunda (TVP) en miembros inferiores en $79 \%$ de los $\operatorname{casos}^{(6)}$ se debe resaltar que la incidencia de TVP ajustada por la edad corresponde a 1.3 casos por 1.000 pacientes-año en hombres y 1.1 casos por 1.000 pacientes-año en mujeres, con mayor riesgo de recurrencia en el paciente de sexo masculino ${ }^{(20-22)}$. Además, la frecuencia de
TVP aumenta en forma proporcional a la edad del paciente, con tasas de 1.8 por cada 1000 personas-año en edades de 65-69 años y 3.1 por cada 1.000 personas-año en pacientes de $85-89$ años ${ }^{(23)}$.

\section{Factores de riesgo. Embolismo pulmonar.}

El embolismo pulmonar se define como un evento clínico multicausal ${ }^{(24,25)}$ que se ha relacionado con condiciones médicas específicas, de las cuales se debe resaltar el cáncer, las enfermedades infecciosas o inflamatorias y la presencia de una TVP o TEP previo ${ }^{(20)}$.

La prevalencia de cáncer en el paciente con TV (TEP o TVP) es de 4 a $20 \%$ en una relación entre variables descrita por Trousseau desde $1868^{(24,26,28)}$. El riesgo (odds ratio; OR) de TV en el paciente con diagnóstico de malignidad es de 4.3 ajustado por edad y sexo (intervalo de confianza, IC 95\%: 3.3-5.6). Las neoplasias hematológicas tienen el mayor riesgo de TV (OR 28; 4-199,7; IC 95\%: 4-199,7) de las cuales el Linfoma No Hodgkin presenta el OR mayor $\left(10,2\right.$; IC 95\%: 1,4-76,9) ${ }^{(25)}$.

Por otro lado, el cáncer de pulmón presenta un odds ratio de TV de 22,2 (IC: 3.6-136.1); las neoplasias gastrointestinales tienen un riesgo similar de TV al compararlo con el grupo anterior (OR 20.3; IC 95\%: 4.9-83) con riesgo mayor en lesiones malignas de intestino delgado (OR 16.4; IC 95\%: 4.2-63.7).

Otras neoplasias como las lesiones primarias de sistema nervioso central (SNC) presentan un riesgo menor a los valores mencionados (OR: 6.7; IC 95\%: 1-45.4) ${ }^{(25)}$.

Cabe mencionar que la presencia de metástasis a distancia en el paciente con neoplasias sólidas aumenta el riesgo hasta cuatro veces de un evento trombótico (TVP o TEP) (OR: 3.9; IC $95 \%: 2.5-6)^{(25)}$.

La incidencia de TV (TEP o TVP) varía según el tipo de neoplasia del paciente, con una tasa general en el paciente con diagnóstico de cáncer de 43 casos por cada 1.000 personas-año; la tasa de incidencia de TV mayor se presenta en las neoplasias cerebrales (116 por cada 1.000 personas-año) y páncreas (102 por cada 1.000 personas-año) al compararla con cáncer de mama 
(21 casos por 1000 personas-año) y próstata (12 casos por cada 1000 personas-año) ${ }^{(29)}$.

La frecuencia de recurrencia anual de TVP posterior a un primer evento es de $5-15 \%$, con una tasa de recurrencia acumulativa de $25 \%$ a los $4 \operatorname{años}^{(20,30)}$. La incidencia de embolismo pulmonar de bajo riesgo asintomática varía entre 39.5 a $49.5 \%$ en el paciente con diagnóstico de TVP proximal ${ }^{(31,32)}$. En el paciente con TEP sintomático idiopático, la recurrencia de embolismo pulmonar es mayor $(17.3 \%)$ que la del paciente con TVP y embolismo pulmonar asintomático $(9.5 \%)^{(33)}$.

El riesgo de embolismo pulmonar en el primer año posterior al un ingreso hospitalario con el diagnóstico de una enfermedad inflamatoria se ha expresado en un estudio de cohorte prospectivo como una incidencia estandarizada de 6.38 (IC: 6.19-6.57); un análisis de subgrupos de este trabajo señala que la incidencia mayor se presenta en los pacientes con miopatía inflamatoria idiopática (polimiositis o dermatomiositis) (incidencia estandarizada 16,44; IC 95\% : 11,57-22,69) ${ }^{(34,35)}$.

La enfermedad inflamatoria intestinal se asocia al desarrollo de TV con una tasa de incidencia de 2.6 casos por cada 1.000 pacientes-año, siendo más alta al documentarse una reagudización durante el internamiento hospitalario (37.5 por cada 1.000 pacientes-año) ${ }^{(36,37)}$. Por otro lado, en el paciente con artritis reumatoide estudios clínicos previos no lograban demostrar una asociación directa de aumento de riesgo de $\mathrm{TV}^{(38)}$; sin embargo, cohortes prospectivas más recientes documentan un evidente aumento del riesgo de trombosis venosa (embolismo pulmonar y/o TVP) en este grupo de pacientes con HR de 1.6 (IC 95\% : 1.4-1.9) a 2 (IC 95\% : $1,9-2,2)^{(39-41)}$.

La granulomatosis con poliangeítis (granulomatosis de Wegener) tiene un riesgo anual de $7 \%$ para desarrollar un episodio de $\mathrm{TV}^{(42)}$; la incidencia de $\mathrm{TV}$ en el paciente con vasculitis ANCA positivo pasa de 1.8 por 1000 personas-año en general a 6,7 casos por cada 1000 personas-año durante la fase activa de la enfermedad $^{(43)}$.

Los procesos infecciosos agudos se encuentran asociados a un aumento de riesgo de TVP y TEP. La tasa de incidencia de TEP en las primeras 2 semanas posterior al diagnóstico de infección del tracto urinario persiste en valores de 2.11 hasta 39 semanas posterior al diagnóstico de la sepsis urinaria. Por otro lado, la presencia de TVP en el paciente con infección de vía respiratoria se asocia a una incidencia de 1.91 en las primeras 2 semanas posterior al diagnóstico de la infección ${ }^{(44,45)}$.

En el paciente sometido a cirugía general, vascular o urológica presenta riesgo de $\mathrm{TV}$ al relacionar el procedimiento quirúrgico con otros factores de riesgo independientes; el embarazo o periodo postparto aumenta el riesgo (OR) a 8.3 ( $\mathrm{p}<0.05$ ), el diagnóstico reciente de sepsis presenta un riesgo (OR) de 4 (p <0.01), la presencia de malignidad tiene un OR de 2.3 (p $<0.01$ ) y la historia de TV previa aumenta 2 veces el riesgo de un nuevo evento trombótico venoso en forma significativa (OR 2.1; $p$ $<0,01)^{(46)}$.

En una cohorte de mujeres hospitalizadas para someterse a cirugía, el riesgo de TV aumenta el riesgo relativo (RR) a 69.1 (IC 95\%: 63.1-75.6) en las primeras 6 semanas postoperatorio y se mantiene hasta las 12 semanas con RR de 19.6 (IC 95\%: 16.6-23.1) El riesgo de TV en esta cohorte es similar para TVP y TEP, siendo el riesgo mayor en las primeras 6 semanas posterior a reemplazo de cadera o de rodilla (RR 220.6; IC $95 \%: 187.8-259.2)^{(47,48)}$.

Por otro lado, la prevalencia de embolismo pulmonar en pacientes posterior a cirugía espinal electiva se ha documentado en $0.06 \%$ (IC 95\%: $0.01-0.12 \%)^{(49)}$. En este tipo de procedimiento, sin una adecuada profilaxis para TVP, las tasas de TV varían según la indicación de la intervención quirúrgica desde $2.3 \%$ en corrección de lesiones degenerativas hasta $6 \%$ en trauma espinal ${ }^{(50)}$.

La necesidad de transfusión se asocia a un aumento del riesgo de TV en el paciente hospitalizado con diagnóstico de cáncer con valores de OR para TV 1.6 (IC95\%: 1.53-1.67) posterior a transfusión de glóbulos rojos empacados y 1.2 (IC95\%: 1.11-1.29) posterior a la infusión de plaquetas endovenosa ${ }^{(51)}$.

Otras variables independientes asociadas a riesgo de TV (TEP y TVP) se han documentado en un estudio de cohorte como el Copenhagen City Heart Study con seguimiento de 19.5 años e 
involucrando 18.954 pacientes. En este trabajo se identificó que el HR de TV es mayor en el hombre (HR 1.24, IC 95\%: 1.08-1.42), pacientes con presión arterial diastólica mayor a 110 $\mathrm{mmHg} \quad$ (HR 1.34; IC95\%: 1.08-1.66), antecedente de tabaquismo (HR 1.52; IC95\%: $1.15-2.01)$ e índice de masa corporal (IMC) $\geq 35$ $\mathrm{kg} / \mathrm{m}^{2}$ (HR 2.1; IC95\%: 1.39-3.16) ${ }^{(52,54)}$. La obesidad es un factor de riesgo independiente para la TV recurrente con incidencia de $17.6 \%$ en el paciente con sobrepeso (índice de masa corporal, IMC $\geq 25 \mathrm{~kg} / \mathrm{m}^{2}$ ) y $18.4 \%$ en el paciente obeso $\left(\overline{\mathrm{IMC}} \geq 30 \mathrm{~kg} / \mathrm{m}^{2}\right)^{(55)}$.

Además de los factores mencionados, otras patologías asociadas a un mayor riesgo de TEP son la insuficiencia cardíaca congestiva, policitemia vera, anemia hemolítica autoinmune, hemoglobinuria paroxística nocturna, anemia drepanocítica, enfermedad pulmonar obstructiva crónica y enfermedad varicosa superficial ${ }^{(56,62)}$.

\section{Diagnóstico. Embolismo Pulmonar.}

El diagnóstico de tromboembolismo pulmonar debe basarse en una estrategia de abordaje clínico basado el grado de probabilidad según hallazgos clínicos, laboratorio y gabinete ${ }^{(63)}$.

Las escalas de validación clínica combinan hallazgos de la historia clínica que han resultado significativos en cohortes de seguimiento en el paciente con sospecha de TEP, tales como hemoptisis o dolor torácico pleurítico $(47 \%$ vs $59 \%$ p $<0.01)$, edema de región sural $(39 \%$ vs $20 \%$, p <0.001), dolor a nivel de región sural $(42 \%$ vs $25 \%, \mathrm{p}<0.001)$ y disnea no complicada (no asociada a hemoptisis, dolor pleurítico o colapso circulatorio) $(36 \% \text { vs } 26 \%, \mathrm{p}<0.01)^{(64)}$.

Por otro lado, los signos clínicos relacionados con aumento de riesgo de embolismo pulmonar incluyen la taquicardia ( $>100$ latidos por minuto; $26 \%$ vs $16 \%$, no significativo), taquipnea ( $>20$ respiraciones por minuto; $57 \%$ vs $47 \%$ no significativo) y otros hallazgos estadísticamente significativos como el aumento del segundo ruido cardíaco $(15 \%$ vs $5 \%, \quad \mathrm{p}<0.001)$, ingurgitación venosa yugular (13\% vs 8\%, p < $0.05)$ y datos al examen físico sugestivos de TVP de miembros inferiores $(47 \%$ vs $23 \%$, p < $0.001)^{(64)}$.

La medición de marcadores de laboratorio como el dímero $\mathrm{D}$ de alta sensibilidad se recomienda para pacientes con baja probabilidad de TEP en las escalas de validación clínica con sensibilidad de $85 \%$ y valor predictivo negativo de $99 \%{ }^{(65-70)}$.

La sensibilidad del dímero D al utilizar la escala de Wells y Geneva es de $84 \%$ y $85 \%$ respectivamente $^{(70)}$. Por la naturaleza estadística del dímero $\mathrm{D}$, al combinar un dímero $\mathrm{D}$ negativo con una escala de validación clínica como la de Wells con un puntaje menor a 4 puntos (baja probabilidad) se puede descartar el diagnóstico de TEP en paciente de atención primaria ambulatorio $^{(71-73)}$. Por el contrario, la gasometría arterial ni la medición del gradiente alvéoloarterial no son de utilidad diagnóstica en el abordaje del paciente con sospecha de embolismo pulmonar ${ }^{(74-80)}$.

A pesar de que en el paciente con TEP se puede encontrar una radiografía de tórax normal, en este estudio se puede identificar algunos hallazgos sugestivos de TEP como la joroba de Hampton y la oligoemia focal (Signo de Westermark) ${ }^{(81)}$.

Las escala clínica de Geneva es de utilidad para el diagnóstico pre-test (antes de estudios de gabinete diagnósticos) del embolismo pulmonar, contempla 8 variables tanto del paciente (edad $>80$ años), historia clínica (cirugía o TVP previo), hallazgos clínicos (taquicardia, hipoxemia o hipocapnia) y hallazgos radiológicos con un puntaje que varía de 0 a 16 puntos; la suma de puntos con valores mayores a 9 presenta una probabilidad pre-test de $73,7 \%$ para TEP en la cohorte de validación ${ }^{(82,87)}$. En la escala de Wells, la presencia de un puntaje mayor a 6 puntos representa un $67 \%$ de riesgo de embolismo pulmonar como paciente de alta probabilidad de $\operatorname{TEP}^{(88,90)}$.

El modelo de Pisa sin complementarse con radiografía de tórax incluye variables negativas para la predicción del riesgo como la historia de enfermedad cardiovascular o pulmonar, ortopnea, fiebre $\left(>38^{\circ} \mathrm{C}\right)$, sibilancias y crépitos, con una prevalencia de TEP de $94 \%$ en el paciente de alta probabilidad clínica ${ }^{(91)}$. Al utilizar la radiografía de tórax como estudio adicional, la prevalencia predicha por el modelo de Pisa es de $98 \%$ en el paciente con alta probabilidad TEP; por esta razón, este modelo de predicción es mucho más preciso que las escalas anteriores ${ }^{(92)}$. Otras escalas como la de Pulmonary Embolism RuleOut Criteria (PERC) tienen un desempeño 
diagnóstico en el paciente con baja probabilidad de TEP menos adecuado ${ }^{(93)}$.

La angiografía pulmonar diagnóstica es el estudio de referencia para el abordaje del paciente con sospecha de $\operatorname{TEP}^{(94)}$. Sin embargo, la necesidad de utilizar un método invasivo en el algoritmo de trabajo diagnóstico hace su uso limitado con una mortalidad de $0.5 \%$ asociada al procedimiento, complicaciones mayores (hipotensión, infarto agudo del miocardio e insuficiencia renal) en $1.5 \%$ y alergia al medio de contraste en $0.1 \%{ }^{(95,96)}$.

Dada la limitación para realizar una angiografía pulmonar a todo paciente con sospecha de TEP, estudios como la tomografía computarizada con angiografía espiral (CTPA) son de utilidad en el diagnóstico de embolismo pulmonar ${ }^{(97,99)}$. Este tipo de tomografía tiene sensibilidad moderadaalta para defectos de perfusión proximal de la vasculatura pulmonar ${ }^{(100,102)}$. La angiografía con tomografía computarizada multidetector (MDCTA) tiene alta sensibilidad y especificidad para el diagnóstico de TEP (83\% sensibilidad; $96 \%$ especificidad $)^{(105,109)}$

La CTPA puede ser de utilidad similar a los estudios de perfusión pulmonar (V/Q Scan $)^{(110)}$. Sin embargo, cerca del $75 \%$ de los pacientes con sospecha de TEP tienen un estudio de perfusión pulmonar V/Q scan no concluyente ${ }^{(111)}$. En el paciente con probabilidad pre-test baja o intermedia un V/Q scan normal excluye la posibilidad diagnóstica de TEP; un estudio de perfusión debe identificar $>2$ defectos segmentarios para el diagnóstico de embolismo pulmonar $^{(110,111)}$.

El Doppler venoso de miembros inferiores no es de utilidad para el diagnóstico de embolismo pulmonar, y un estudio que no encuentre TVP no descarta la presencia de $\mathrm{TEP}^{(112-113)}$. Por otro lado, la combinación de CTPA negativo por defectos de perfusión $\mathrm{y}$ dímero $\mathrm{D}$ negativo descarta la presencia de TEP sin la necesidad de el estudio de la extremidad inferior con ultrasonido $^{(114,118)}$.

Otros estudios como la resonancia magnética con gadolinio tienen alta especificidad en el paciente con sospecha de embolismo pulmonar pero presenta limitaciones técnicas hasta en $52 \%$ de los casos valorados ${ }^{(119,120)}$. Asimismo los hallazgos electrocardiográficos como la inversión de la onda $\mathrm{T}$ en V1-V4, patrón QR en la D I, bloqueo de rama derecha completo o incompleto y el patrón S (I) Q (III) T (III) tienen un valor limitado en el diagnóstico de $\operatorname{TEP}^{(121,122)}$.

\section{CONCLUSIONES}

El TEP es un evento clínico frecuente, asociado a importante morbi-mortalidad según la presentación y características del paciente afectado.

Presenta una etiología multicausal y los postulados de Virchow aún son vigentes en su fisiopatología. Múltiples factores de riesgo han sido asociados en distinto grado al TEP donde destacan el cáncer, las enfermedades infecciosas e inflamatorias, trombosis previas, entre otros.

$\mathrm{Su}$ diagnóstico debe basarse en una estrategia clínica lógica, individualizando al paciente según su grado de probabilidad de TEP tomando en cuenta los hallazgos clínicos, de laboratorio y gabinete, analizando las diferentes ventajas y desventajas de cada una de estas herramientas diagnósticas.

\section{REFERENCIAS BIBLIOGRÁFICAS}

1. Jaff MR McMurtry MS Archer SL et al. Management of massive and submassive pulmonary embolism, iliofemoral deep vein thrombosis, and chronic thromboembolic pulmonary hypertension: a scientific statement from the American Heart Association. Circulation. 2011; 123:1788-1830.

2. Kucher N Rossi E De Rosa M Goldhaber SZ. Massive pulmonary embolism. Circulation. 2006;113:577-582.

3. Kasper W Konstantinides S Geibel A et al. Management strategies and determinants of outcome in acute major pulmonary embolism: results of a multicenter registry. J Am Coll Cardiol. 1997;30: 1165-1171.

4. Virchow R. (1855) Archiv fuer pathologische Anatomie und Physiologie und fuer klinische Medizin, 8, 23, [Traducción en: Ackerknecht, E.H. Rudolf Virchow. Doctor, Statesman, Anthropologist. Madison, University of Wisconsin, 1953, pp 83]. 
5. Nicolaides AN Kakkar VV Field ES Renney JT. The origin of deep vein thrombosis: a venographic study. $\mathrm{Br} \mathrm{J}$ Radiol. 1971;44: 653-663.

6. Tapson V. Acute pulmonary embolism. N Engl J Med. 2008;358:1037-1052.

7. Killewich LA Bedford GR Beach KW Strandness D. Spontaneous lysis of deep venous thrombi: rate and outcome. J Vasc Surg. 1989;9:89-97.

8. Heit JA Melton LJ Lohse CM et al. Incidence of venous thromboembolism in hospitalized patients vs community residents. Mayo Clin Proc. 2001;76(11): 1102-1110.

9. Huerta C Johansson S Wallander MA García LA. Risk factors and short-term mortality of venous thromboembolism diagnosed in the primary care setting in the United Kingdom. Arch Intern Med. 2007;14;167(9):935-943.

10. Oger E. Incidence of venous thromboembolism: a community-based study in Western France. EPI-GETBP Study Group. Groupe d'Etude de la Thrombose de Bretagne Occidentale. Thromb Haemost. 2000;83(5):657-660.

11. Jang MJ Bang SM Oh D. Incidence of venous thromboembolism in Korea: from the Health Insurance Review and Assessment Service database. J Thromb Haemost. 2011;9(1):85-91.

12. Molina JA Jiang ZG Heng BH Ong BK. Venous thromboembolism at the $\mathrm{Na}$ tional Healthcare Group, Singapore. Ann Acad Med Singapore. 2009;38(6): 470-478.

13. Stein PD Beemath A Olson RE. Trends in the incidence of pulmonary embolism and deep venous thrombosis in hospitalized patients. Am J Cardiol. 2005;95 (12):1525-1526.

14. Yang Y Liang L Zhai Z et al. Pulmonary embolism incidence and fatality trends in Chinese hospitals from 1997 to 2008: a multicenter registration study. PLoS One. 2011;6(11):e26861

15. Kuklina EV Meikle SF Jamieson DJ. Severe obstetric morbidity in the United States: 1998-2005. Obstet Gynecol. 2009;113(2):293-299.

16. Silverstein MD Heit JA Mohr DN et al. Trends in the incidence of deep vein thrombosis and pulmonary embolism: a 25-year population-based study. Arch
Intern Med. 1998;158:585-593.

17. Heit JA Cohen AT Anderson FA. VTE Impact Assessment Group. Estimated annual number of incident and recurrent, non-fatal and fatal venous thromboembolism (VTE) events in the U.S. Blood 2005; 106:267a. abstract.

18. Hoffmann B Gross CR Jöckel KH Kröger K. Trends in mortality of pulmonary embolism: an international comparison. Thromb Res. 2010;125(4): 303-308.

19. Kyrle PA Eichinger S. Deep vein thrombosis. Lancet. 2005;365(9465): 1163-1174.

20. Cushman M Tsai AW White RH et al. Deep vein thrombosis and pulmonary embolism in two cohorts: the longitudinal investigation of thromboembolism etiology. Am J Med 2004;117:19-25.

21. Kyrle PA Minar E Bialonczyk C Hirschl M Weltermann A Eichinger S. The risk of recurrent venous thromboembolism in men and women. N Engl J Med. 2004;350:2558-2563.

22. Kniffin WD Baron JA Barrett J Birkmeyer JD Anderson FA. The epidemiology of diagnosed pulmonary embolism and deep venous thrombosis in the elderly. Arch Intern Med. 1994;154:861866.

23. Blom JW Doggen CJ Osanto S Rosendaal FR. Malignancies, prothrombotic mutations, and the risk of venous thrombosis. JAMA. 2005;293:715-722.

24. Rosendaal FR. Venous thrombosis: a multicausal disease. Lancet. 1999;353: 1167-1173.

25. Trousseau A. Phlegmasia Alba Dolens: Lectures on Clinical Medicine. London, England: The New Sydenham Society. 1868;5:281-331.

26. Baron JA Gridley G Weiderpass E Nyren O Linet M. Venous thromboembolism and cancer. Lancet. 1998; 351:1077-1080.

27. Heit JA Silverstein MD Mohr DN Petterson TM O'Fallon WM Melton LJ. Risk factors for deep vein thrombosis and pulmonary embolism: a populationbased case-control study. Arch Intern Med. 2000;160:809-815.

28. Horsted F West J Grainge MJ. Risk of venous thromboembolism in patients with cancer: a systematic review and 
meta-analysis. "Risk of Venous Thromboembolism in Patients with Cancer: PLoS Medicine 9.7 (2012): e1001275.

29. Prandoni P Lensing AW Cogo A et al. The long-term clinical course of acute deep venous thrombosis. Ann Intern Med. 1996;125:1-7.

30. Meignan M Rosso J Gauthier $\mathrm{H}$ et al. Systematic lung scans reveal a high frequency of silent pulmonary embolism in patients with proximal deep venous thrombosis. Arch Intern Med. 2000;24;160(2):159-164.

31. Eichinger S Weltermann A Minar E et al. Symptomatic pulmonary embolism and the risk of recurrent venous thromboembolism. Arch Intern Med. 2004; 12;164(1):92-96.

32. Zöller B Li X Sundquist J Sundquist K. Risk of pulmonary embolism in patients with autoimmune disorders: a nationwide follow-up study from Sweden. Lancet. 2012;21;379(9812):244-249.

33. Grainge MJ West J Card TR. Venous thromboembolism during active disease and remission in inflammatory bowel disease: a cohort study. Lancet. 2010; 375(9715):657-663.

34. Miehsler W Reinisch W Valic E et al. Is inflammatory bowel disease an independent and disease specific risk factor for thromboembolism? Gut. 2004;53(4): 542-548.

35. Holmqvist ME Neovius M Eriksson $\mathrm{J}$ et al. Risk of venous thromboembolism in patients with rheumatoid arthritis and association with disease duration and hospitalization. JAMA. 2012;308(13): 1350-1356.

36. Kim SC Schneeweiss S Liu J Solomon $\mathrm{DH}$. Risk of venous thromboembolism in patients with rheumatoid arthritis. Arthritis Care Res (Hoboken). 2013;65 (10):1600-1607.

http://www.ncbi.nlm.nih.gov.ezproxy.si bdi.ucr.ac.cr:2048/entrez/query.fcgi?cm $\mathrm{d}=$ Retrieve $\& \mathrm{db}=$ pubmed $\&$ dop $\mathrm{t}=$ Abstra ct\&list $\% 5$ Fuids $=23032551 \&$

37. Bacani AK Gabriel SE Crowson CS Heit JA Matteson EL. Non-cardiac vascular disease in rheumatoid arthritis: increase in venous thromboembolic events? Arthritis Rheum. 2012;64(1): 53-61.

38. Merkel PA Lo GH Holbrook JT et al.
Brief communication: high incidence of venous thrombotic events among patients with Wegener granulomatosis: the Wegener's Clinical Occurrence of Thrombosis (WeCLOT) Study. Ann Intern Med. 2005;19:(8):620-626.

39. Stassen PM Derks RP Kallenberg CG Stegeman CA. Venous thromboembolism in ANCA-associated vasculitis-incidence and risk factors. Rheumatology (Oxford). 2008;47(4):530-534.

40. Smeeth L Cook C Thomas S Hall AJ Hubbard R Vallance P. Risk of deep vein thrombosis and pulmonary embolism after acute infection in a community setting. Lancet. 2006;367(9516): 1075-1079.

41. Neumann V O'Connor RJ Bhakta BB Tennant A. DVT and pulmonary embolism after acute infection. Lancet. 2006; 368(9531):201.

42. Bahl V Hu HM Henke PK Wakefield TW Campbell DA Jr Caprini JA. A validation study of a retrospective venous thromboembolism risk scoring method. Ann Surg. 2010;251(2):344-350.

43. Sweetland S Green J Liu B et al. Duration and magnitude of the postoperative risk of venous thromboembolism in middle aged women: prospective cohort study. BMJ. 2009;339:b4583.

44. Cohen AT. Prevention of postoperative venous thromboembolism. BMJ. 2009; 339:b4477

45. Sansone JM del Rio AM Anderson PA. The prevalence of and specific risk factors for venous thromboembolic disease following elective spine surgery. Joint Surg Am. 2010;92(2):304-313.

46. Cheng JS Arnold PM Anderson PA Fischer D Dettori JR. Anticoagulation risk in spine surgery. Spine (Phila $\mathrm{Pa}$ 1976). 2010;35(9 Suppl):S117-S124.

47. Khorana AA Francis CW Blumberg N Culakova E Refaai MA Lyman GH. Blood transfusions, thrombosis, and mortality in hospitalized patients with cancer. Arch Intern Med. 2008;168(21): 2377-2381.

48. Holst AG Jensen G Prescott E. Riskfactors for venous thromboembolism: results from the Copenhagen City Heart Study. Circulation. 2010;121(17):18961903.

49. Hansson PO Eriksson H Welin L Svärd- 
sudd K Wilhelmsen L. Smoking and abdominal obesity: risk factors for venous thromboembolism among middleaged men: "the study of men born in 1913”. Arch Intern Med. 1999;159(16): 1886-1890.

50. Douketis J Tosetto A Marcucci M et al. Risk of recurrence after venous thromboembolism in men and women: patient level meta-analysis. BMJ. 2011;342: $\mathrm{d} 813$.

51. Eichinger S Hron G Bialonczyk C et al. Overweight, obesity, and the risk of recurrent venous thromboembolism. Arch Intern Med. 2008;168(15):1678-1683.

52. Piazza G Goldhaber SZ. Pulmonary embolism in heart failure. Circulation. 2008;118(15):1598-1601.

53. Landolfi R Cipriani MC Novarese L. Thrombosis and bleeding in polycythemia vera and essential thrombocythemia: pathogenetic mechanisms and prevention. Best Pract Res Clin Haematol. 2006;19(3):617-633.

54. Hoffman PC. Immune hemolytic anemia-selected topics. Hematology Am Soc Hematol Educ Program. 2009:8086.

55. Ray JG Burows RF Ginsberg JS Burrows EA. Paroxysmal nocturnal hemoglobinuria and the risk of venous thrombosis: review and recommendations for management of the pregnant and non-pregnant patient. Haemostasis. 2000;30(3):103-117.

56. Austin H Key NS Benson JM et al. Sickle cell trait and the risk of venous thromboembolism among blacks. Epub 2007 Apr 4.

57. Tapson VF. The role of smoking in coagulation and thromboembolism in chronic obstructive pulmonary disease. Proc Am Thorac Soc. 2005;2(1):71-77.

58. Torbicki A Perrier A Konstantinides S et al. Guidelines on the diagnosis and management of acute pulmonary embolism: the Task Force for the Diagnosis and Management of Acute Pulmonary Embolism of the European Society of Cardiology (ESC). Eur Heart J. 2008;29(18):2276-2315.

59. Lucassen W Geersing GJ Erkens PM et al. Clinical decision rules for excluding pulmonary embolism: a meta-analysis. Ann Intern Med. 2011;155(7):448-460.
60. Stein PD Beemath A Matta F et al. Clinical characteristics of patients with acute pulmonary embolism: data from PIOPED II. Am J Med. 2007;120(10): 871-879.

61. Qaseem A Snow V Barry P et al. Current diagnosis of venous thromboembolism in primary care: a clinical practice guideline from the American Academy of Family Physicians and the American College of Physicians. Ann Fam Med. 2007;5(1):57-62.

62. Segal JB Eng J Tamariz LJ Bass EB. Review of the evidence on diagnosis of deep venous thrombosis and pulmonary embolism. Ann Fam Med. 2007;5(1):6373.

63. Qaseem A Snow V Barry P et al. Current diagnosis of venous thromboembolism in primary care: a clinical practice guideline from the American Academy of Family Physicians and the American College of Physicians. Ann Intern Med. 2007;146(6):454-458.

64. Campos-Outcalt D. New guidelines on DVT and pulmonary embolism. Am Fam Physician. 2007;76(8):1116.

65. Chapman NH Brighton $\mathrm{T}$ Harris $\mathrm{MF}$ Caplan GA Braithwaite J Chong $\mathrm{BH}$. Venous thromboembolism - management in general practice. Aust Fam Physician. 2009;38(1-2):36-40.

66. Lucassen W Geersing GJ Erkens PM et al. Clinical decision rules for excluding pulmonary embolism: a meta-analysis. Ann Intern Med. 2011;155(7):448-460.

67. Geersing GJ Erkens PM Lucassen WA et al. Safe exclusion of pulmonary embolism using the Wells rule and qualitative D-dimer testing in primary care: prospective cohort study. BMJ. 2012;345:e6564.

68. Kruip MJ Leclercq MG van der Heul C Prins MH Büller HR. Diagnostic strategies for excluding pulmonary embolism in clinical outcome studies. A systematic review. Ann Intern Med. 2003;138(12): 941-951.

69. Jacobs LG. When are you sure that you are wrong? How to exclude a pulmonary embolism. J Am Geriatr Soc. 2004; 52(2):311-313.

70. Rodger MA Carrier M Jones GN et al. Diagnostic value of arterial blood gas measurement in suspected pulmonary 
embolism. Am J Respir Crit Care Med. 2000;162(6):2105-2108.

71. Stein PD Terrin ML Hales CA et al. Clinical, laboratory, roentgenographic, and electrocardiographic findings in patients with acute pulmonary embolism and no pre-existing cardiac or pulmonary disease. Chest. 1991;100(3):598603.

72. Tapson VF. Pulmonary embolism: the diagnostic repertoire. Chest. 1997;112 (3):578-580.

73. Stein PD Goldhaber SZ Henry JW. Alveolar-arterial oxygen gradient in the assessment of acute pulmonary embolism. Chest. 1995;107(1):139-143.

74. Stein PD Goldhaber SZ Henry JW Miller AC. Arterial blood gas analysis in the assessment of suspected acute pulmonary embolism. Chest. 1996;109(1): 78-81.

75. Egermayer P. Normoxemia and pulmonary embolism. Chest. 2001;120(5): 1756.

76. Deutsch AB Twitty P Downes K Parsons MT. Assessment of the alveolararterial oxygen gradient as a screening test for pulmonary embolism in pregnancy. Am J Obstet Gynecol. 2010;203 (4):373.e1-4.

77. Tapson VF Carroll BA Davidson BL et al. The diagnostic approach to acute venous thromboembolism. Clinical practice guideline. American Thoracic Society. Am J Respir Crit Care Med. 1999;160(3):1043-1066

78. Rodger MA Carrier M Jones GN et al. Diagnostic value of arterial blood gas measurement in suspected pulmonary embolism. Am J Respir Crit Care Med. 2000;162(6):2105-2108.

79. Wicki J Perneger TV Junod AF Bounameaux H Perrier A. Assessing clinical probability of pulmonary embolism in the emergency ward: a simple score. Arch Intern Med. 2001;161(1): 92-97.

80. Jay SJ. Is smoking a risk factor for pulmonary embolism? Arch Intern Med. 2001;161(15):1925-1926.

81. Le Gal G Righini M Roy PM et al. Prediction of pulmonary embolism in the emergency department: the revised Geneva score. Ann Intern Med. 2006; 144(3):165-171.
82. Sox HC. Better care for patients with suspected pulmonary embolism. Ann Intern Med. 2006;144(3):210-212.

83. Dunn A. The revised Geneva score predicted pulmonary embolism in patients with shortness of breath or chest pain. ACP J Club. 2006;145(1):25.

84. Douma RA Mos IC Erkens PM et al. Performance of 4 clinical decision rules in the diagnostic management of acute pulmonary embolism: a prospective cohort study. Ann Intern Med. 2011;154 (11):709-718.

85. Yap KS Kalff V Turlakow A Kelly MJ. A prospective reassessment of the utility of the Wells score in identifying pulmonary embolism. Med J Aust. 2007;187 (6):333-336.

86. Biss TT Brandão LR Kahr WH Chan AK Williams S. Clinical probability score and D-dimer estimation lack utility in the diagnosis of childhood pulmonary embolism. J Thromb Haemost. 2009;7(10):1633-1638.

87. Miniati M Bottai M Monti S Salvadori M Serasini L Passera M. Simple and accurate prediction of the clinical probability of pulmonary embolism. Am J Respir Crit Care Med. 2008;178(3):290294.

88. Miniati M Bottai M Monti S. Comparison of 3 clinical models for predicting the probability of pulmonary embolism. Medicine (Baltimore). 2005;84(2):107114.

89. Singh B Parsaik AK Agarwal D Surana A Mascarenhas SS Chandra S. Diagnostic accuracy of pulmonary embolism rule-out criteria: a systematic review and meta-analysis. Ann Emerg Med. 2012;59(6):517-520.

90. Roy PM Colombet I Durieux P Chatellier G Sors H Meyer G. Systematic review and meta-analysis of strategies for the diagnosis of suspected pulmonary embolism. BMJ. 2005;331 (7511):259.

91. Stein PD Athanasoulis C Alavi A et al. Complications and validity of pulmonary angiography in acute pulmonary embolism. Circulation. 1992;85(2):462468.

92. Singh H Cardella JF Cole PE et al. Quality improvement guidelines for diagnostic arteriography. J Vasc Interv 
Radiol. 2002;13(1):1-6.

93. Moores LK Jackson WL Jr Shorr AF Jackson JL. Meta-analysis: outcomes in patients with suspected pulmonary embolism managed with computed tomographic pulmonary angiography. Ann Intern Med. 2004;141(11):866-874.

94. Frost SD. Defining the role of computed tomographic pulmonary angiography in suspected pulmonary embolism. Ann Intern Med. 2005;142(9):800-801.

95. Mos IC Klok FA Kroft LJ DE Roos A Dekkers OM Huisman MV. Safety of ruling out acute pulmonary embolism by normal computed tomography pulmonary angiography in patients with an indication for computed tomography: systematic review and meta-analysis. $\mathrm{J}$ Thromb Haemost. 2009;7(9):14911498.

96. Quiroz R Kucher N Zou KH et al. Clinical validity of a negative computed tomography scan in patients with suspected pulmonary embolism: a systematic review. JAMA. 2005;293(16):20122017.

97. Stein PD Kayali F Hull RD. Spiral computed tomography for the diagnosis of acute pulmonary embolism. Thromb Haemost. 2007;98(4):713-720.

98. Mullins MD Becker DM Hagspiel KD Philbrick JT. The role of spiral volumetric computed tomography in the diagnosis of pulmonary embolism. Arch Intern Med. 2000;160(3):293-298.

99. Kane KY Ellis MR. Is spiral (helical) computed tomography useful for diagnosing pulmonary embolism? J Fam Pract. 2000;49(5):467-468.

100. Kelly MJ Pitman A. Defining the role of helical computerised tomography (CT) in the diagnosis of pulmonary embolism (PE). Aust N Z J Med. 2000;30(2):191193.

101. Stein PD Fowler SE Goodman LR et al. Multidetector computed tomography for acute pulmonary embolism. $\mathrm{N}$ Engl J Med. 2006;354(22):2317-2327.

102. Brotman DJ. Computed tomography for pulmonary embolism. N Engl J Med. 2006;355(9):955.

103. Cronin P Weg JG Kazerooni EA. The role of multidetector computed tomography angiography for the diagnosis of pulmonary embolism. Semin Nucl Med.
2008;38(6):418-431.

104.Perrier A. Multidetector CTA with venography was more sensitive for diagnosing pulmonary embolism than CTA alone. ACP J Club. 2006;145(3):76.

105.Perrier A Bounameaux H. Accuracy or outcome in suspected pulmonary embolism. N Engl J Med. 2006;354(22): 2383-2385.

106.Kaatz S Buckley J. Review: helical CT has better discriminatory power than ventilation-perfusion scan to exclude pulmonary embolism. ACP J Club. 2005;143(2):52.

107.Hayashino Y Goto M Noguchi Y Fukui T. Ventilation-perfusion scanning and helical CT in suspected pulmonary embolism: meta-analysis of diagnostic performance. Radiology. 2005;234(3):740748 .

108. Turkstra F Kuijer PM van Beek EJ Brandjes DP ten Cate JW Büller HR. Diagnostic utility of ultrasonography of leg veins in patients suspected of having pulmonary embolism. Ann Intern Med. 1997;126(10):775-781.

109. Sheiman RG McArdle CR. Clinically suspected pulmonary embolism: use of bilateral lower extremity US as the initial examination--a prospective study. Radiology. 1999;212(1):75-78.

110.Perrier A Roy PM Sánchez O et al. Multidetector-row computed tomography in suspected pulmonary embolism. $\mathrm{N}$ Engl J Med. 2005;352(17):17601768.

111. Goldhaber SZ. Multislice computed tomography for pulmonary embolism: a technological marvel. N Engl J Med. 2005;352(17):1812-1814.

112. Turpie AG. Multidetector-row computed tomography in suspected pulmonary embolism. N Engl J Med. 2005;353(6): 630-631.

113.Le Gal G Righini M Mottier D. Clinical diagnosis of pulmonary embolism: a real challenge. Rev Med Interne. 2007;28 (6):394-399.

114. Stein PD Gottschalk A Sostman HD. Methods of Prospective Investigation of Pulmonary Embolism Diagnosis III (PIOPED III). Semin Nucl Med. 2008;38(6):462-470.

115. Stein PD Chenevert TL Fowler SE et al. Gadolinium-enhanced magnetic reso- 
nance angiography for pulmonary embolism: a multicenter prospective study (PIOPED III). Ann Intern Med. 2010; 152(7):434-443.

116. Davidson BL Lacrampe MJ. Why can't magnetic resonance imaging reliably diagnose pulmonary embolism? Ann Intern Med. 2010;152(7):467-468.

117.Brown G Hogg K. Best evidence topic report. Diagnostic utility of electrocardiogram for diagnosing pulmonary embolism. Emerg Med J. 2005;22(10):729730.

118.Harrigan RA Jones K. ABC of clinical electrocardiography. Conditions affecting the right side of the heart. BMJ. 2002;324(7347):1201-1204.

119. Ryan D Hong T Bardeesy N. Pancreatic Adenocarcinoma. N Engl J Med 2014;371:1039-1049.

120.Simard E Ward E Siegel R Jemal A. Cancers With Increasing Incidence Trends in the United States: 1999 Through 2008. CA Cancer J Clin. 2012; 62:118-128.

121.Krejs G. Pancreatic Cancer: Epidemiology and Risk Factors. Dig Dis 2010; 28:355-358.

122.Habib M Wasif Saif M. Thromboembolism and Anticoagulation in Pancreatic Cancer. JOP. J Pancreas (Online) 2013; 14(2):135-137.

\section{DECLARACIÓN DE CONFLICTO DE IN- TERESES}

El autor declara que no existen conflictos de interés. 